\section{Distribuição espacial da mortalidade por infarto agudo do miocárdio no Município do Rio de Janeiro, Brasil}

\author{
Spatial distribution of mortality from acute \\ myocardial infarction in Rio de Janeiro, Brazil
}

\author{
1 Escola de Enfermagem \\ Alfredo Pinto, Universidade \\ Federal do Estado do \\ Rio de Janeiro, \\ Rio de Janeiro, Brasil. \\ 2 Escola Nacional de Saúde \\ Pública Sergio Arouca, \\ Fundação Oswaldo Cruz, \\ Rio de Janeiro, Brasil. \\ 3 Centro de Informação \\ Científica e Tecnológica, \\ Fundação Oswaldo Cruz, \\ Rio de Janeiro, Brasil. \\ Correspondência \\ E. C. P. Melo \\ Departamento de \\ Enfermagem em Saúde \\ Pública, Escola de \\ Enfermagem Alfredo Pinto, \\ Universidade Federal do \\ Estado do Rio de Janeiro. \\ Rua Barão de Mesquita 850, \\ Bloco D, apto. 406, \\ Rio de Janeiro, $R J$ \\ 20540-004, Brasil. \\ enirtes@globo.com
}

\begin{abstract}
The study analyzes the spatial distribution of mortality from acute myocardial infarction (AMI) in Rio de Janeiro, Brazil. Data from the Mortality Information System refers to the year 2000. Empirical Bayes smoothing technique was used to minimize random variation in mortality coefficients due to the population size in the geographic analytical units. Spatial distribution of AMI mortality in the city of Rio de Janeiro is heterogeneous and displays a pattern associated with a strong socioeconomic gradient. The decreased AMI risk in the West Side of the city fails to reflect the social inequality and limited access to healthcare services observed there. A more likely hypothesis is that the risk of AMI death was underestimated in the West Side, due to the high proportion of ill-defined causes of death in that region. In the rest of the city, the spatial pattern of AMI mortality showed higher values in poorer areas. The various hospitals treating AMI also show a clear pattern in their areas of influence.
\end{abstract}

Myocardial Infarction; Spatial Analysis; Information Systems; Health Services Accessibility
Enirtes Caetano Prates Melo 1

Marilia Sá Carvalho ${ }^{2}$

Claudia Travassos 3

\section{Introdução}

Na maioria dos países a doença isquêmica do coração representa uma das principais causas de óbito em homens e mulheres acima de trinta anos de idade. Apesar da tendência de declínio observada a partir da década de 80 em vários países, a mortalidade por doença isquêmica permanece elevada no Brasil 1,2. Cabe destaque a elevada proporção de óbitos precoces por doença isquêmica do coração no Brasil, muito superior a de países como Estados Unidos, Cuba, Argentina, Inglaterra, Portugal, Espanha, França, Dinamarca e Japão 3,4,5,6.

Vários estudos, voltados para as variações geográficas da doença isquêmica do coração, têm mostrado a importância da inclusão dos fatores ambientais relacionados a esse agravo para a compreensão de como o contexto afeta a saúde de grupos populacionais 7,8. Dentre os fatores ambientais que estão relacionados à doença isquêmica do coração cabe destacar o papel do desenvolvimento sócio-econômico, do processo de urbanização e seu impacto sobre o estilo de vida das populações 9 .

A distribuição da morbidade e mortalidade por doença isquêmica do coração expressa diferenças sociais associadas à renda, escolaridade, ocupação e classe social 10,11,12. O declínio específico da mortalidade iniciou-se nos estratos da população com padrão sócio-econômico mais elevado 13. Áreas com condições desfavo- 
ráveis apresentam uma sobrevida mais baixa 9 . A condição econômica diminui o acesso ao tratamento, especialmente a procedimentos como angioplastia e cirurgia de revascularização do miocárdio 12,14,15.

Vários estudos realizados no Rio de Janeiro indicam que populações socialmente desfavorecidas apresentam índices de mortalidade mais altos para diversas causas de óbito 16,17. Pesquisa ecológica realizada no Município do Rio de Janeiro por Szwarcwald et al. 18 examina padrões geográficos de alguns indicadores de resultado (taxa de mortalidade padronizada, taxa de homicídio, taxa de mortalidade infantil), relacionando-os a indicadores demográficos e sócio-econômicos. Os achados dessa pesquisa evidenciam a influência contextual das características sociais e de organização do local de residência sobre a saúde. Ao analisar as relações entre os padrões espaciais de mortalidade e o perfil sócio-econômico dos bairros do Rio de Janeiro, Santos \& Noronha 19 observaram que as maiores diferenças entre os estratos sócio-econômicos ocorreram na mortalidade por doenças circulatórias, causas mal definidas e causas externas. Estudo de Souza 2 confirma a correlação positiva, na população de 30 a 70 anos, entre renda e mortalidade por doença isquêmica do coração nos bairros da cidade.

O Município do Rio de Janeiro apresenta uma das mais elevadas taxas de mortalidade por infarto agudo do miocárdio do país, estando atrás apenas de Porto Alegre. O infarto é um evento agudo, que requer atendimento médico e rapidez na confirmação do diagnóstico e instituição do tratamento. Mais de $50 \%$ dos óbitos ocorrem na primeira hora de evolução 20 . Pacientes tratados dentro desse período de tempo experimentam uma redução significativa da mortalidade hospitalar.

Localizar eventos de saúde nos bairros desse município pode permitir a identificação de associações entre essa diversidade geográfica, os padrões de mortalidade e o acesso aos serviços de saúde. A visualização dessas relações no território vem contribuir para a formulação de políticas públicas mais integradas e efetivas. Este estudo tem como objetivo analisar a distribuição espacial da mortalidade por infarto agudo do miocárdio nos bairros do Município do Rio de Janeiro, buscando identificar áreas de sobrerisco e a relação espacial dos óbitos com a distribuição dos serviços de saúde.

\section{Métodos}

Este é um estudo ecológico que considera como unidades de análise os 158 bairros do $\mathrm{Mu}$ nicípio do Rio de Janeiro, que em 2000 contava com uma população de 5.857 .895 pessoas.

Foram utilizadas as bases de dados referentes ao Sub-sistema de Informações sobre Mortalidade (SIM), disponibilizadas pela Secretaria Municipal de Saúde do Rio de Janeiro (SMS-RJ) após aprovação do estudo pelo Comitê de Ética em Pesquisa da Fundação Oswaldo Cruz (FIOCRUZ). As informações sobre a população residente, por sexo, faixa etária e renda, foram obtidas por meio do Censo Demográfico de 2000, realizado pelo Instituto Brasileiro de Geografia e Estatística 21. Foram utilizadas também as bases digitais geradas no Laboratório de Geoprocessamento do Departamento de Informações em Saúde, Centro de Informação Científica e Tecnológica (DIS/CICT/FIOCRUZ). O georreferenciamento dos óbitos foi efetuado com base na variável bairro de residência, que consta na declaração de óbito.

Foram analisados os óbitos cuja causa básica foi infarto agudo do miocárdio (código I21 da Classificação Internacional de Doenças e Problemas Relacionados à Saúde, 10a RevisãoCID-10), ocorridos no Município do Rio de Janeiro durante o ano 2000. Incluiu-se no estudo apenas os óbitos de pessoas em que o Rio de Janeiro foi o município de residência. Dos 4.015 óbitos por infarto agudo do miocárdio registrados em 2000, 3.651 atendiam aos critérios estabelecidos. Excluídos os óbitos com endereço ignorado (0,5\%), estudou-se 3.633 óbitos por infarto agudo do miocárdio.

Em decorrência do grande crescimento dos óbitos por causas mal definidas nesse município, a partir de 1990, e considerando a hipótese de subenumeração de óbitos por infarto agudo do miocárdio no SIM, incluiu-se na análise estes óbitos, classificados no capítulo XVIII (R00 a R99) da CID-10. Esse capítulo inclui sintomas, sinais e achados anormais de exames clínicos ou de outros procedimentos de investigação diagnóstica, além de afecções mal definidas para as quais não haja um diagnóstico classificado em outra parte. Durante o ano 2000, o SIM registrou no Município do Rio de Janeiro 5.304 óbitos por causas mal definidas.

As variáveis analisadas da Declaração de Óbito (DO) foram: sexo, idade, bairro de residência, local de ocorrência do óbito (hospital, outros estabelecimentos de saúde que prestam assistência individual ou coletiva, domicílio, via pública, outros) e o estabelecimento de saúde onde ocorreu o óbito. 
Trabalhou-se com duas estratégias que serviram de base para a construção dos mapas da distribuição espacial dos óbitos por infarto agudo do miocárdio. Primeiro utilizou-se a taxa específica de mortalidade e a razão de mortalidade padronizada (RMP) por idade e sexo na comparação entre os bairros. Os coeficientes de mortalidade foram ajustados por meio do método indireto, usando-se a população geral do Município do Rio de Janeiro como padrão.

Segundo, considerando que existe uma variabilidade aleatória da RMP associada ao tamanho das unidades geográficas de análise (bairros), utilizou-se o modelo bayesiano empírico de suavização local a fim de minimizar essa variação ${ }^{22}$. Esse método, ao estimar o risco de uma pequena área, tem como idéia central o uso de informações de áreas vizinhas que compõem a região sob estudo, reduzindo assim a flutuação aleatória dos coeficientes. A matriz de vizinhança foi definida por adjacências. Os bairros tiveram suas taxas reestimadas aplicando-se uma média ponderada entre o valor medido e a taxa média global, em que o peso da média é inversamente proporcional à população da região.

É razoável supor que as taxas das diferentes regiões estão autocorrelacionadas, e levar em conta o comportamento dos vizinhos para estimar uma taxa mais realista para as regiões de menor população. Esta formulação sugere o uso de técnicas de estimação bayesiana. Nesse contexto, considera-se que a taxa "real" $\theta_{i}$ associada a cada área não é conhecida, e dispomos de uma taxa observada $t_{i}=z_{i} / n_{i}$, onde $n_{i}$ é o número de pessoas observadas, e $z_{i}$ é o número de eventos na i-ésima área. As regiões terão suas taxas reestimadas aplicando-se uma média ponderada entre o valor medido e a taxa média global, em que o peso da média será inversamente proporcional à população da região.

Utilizou-se o índice de Moran I sobre as taxas não suavizadas para testar a existência de autocorrelação espacial (tendência, cluster e/ ou regularidade). Esse índice permite testar se áreas vizinhas apresentam maior semelhança na mortalidade observada em comparação com aquele que seria esperado em um padrão de completa aleatoriedade espacial 23 . Os valores deste teste encontram-se entre - 1 e 1 , que quantifica a autocorrelação existente entre as áreas. Utilizou-se como critério de significância o valor de p abaixo de 0,05.

Nos mapas que apresentam a razão de mortalidade foram definidas nove classes com base nas variáveis: razão de mortalidade por infarto agudo do miocárdio não padronizada, RMP por idade e sexo, RMP segundo local de ocorrência do óbito, RMP segundo natureza jurídica do hospital onde ocorreu o óbito (privado, municipal, estadual, federal, contratado, filantrópico). Uma RMP abaixo de 1 significa baixa mortalidade; se a razão resulta em um número acima de 1, a mortalidade é elevada. Um valor igual à média indica mortalidade idêntica nos bairros da cidade. Os mapas obedeceram a uma escala cromática em tons de cinza; áreas com uma RMP situada em torno de zero $(0,75$ a 1,25$)$ foram deixadas em branco. Os mapas apresentados foram submetidos à correção usando-se o modelo bayesiano empírico de suavização.

Utilizando-se as bases cedidas pelo Laboratório de Geoprocessamento do DIS/CICT/FIOCRUZ foi possível visualizar geograficamente os hospitais que apresentaram, durante 2000, um volume igual ou superior a dez óbitos por infarto agudo do miocárdio. Após a classificação desses hospitais segundo natureza jurídica, elaborou-se uma segunda camada nos mapas temáticos por bairros localizando-os.

Os bairros que compõem o município estão distribuídos em dez áreas de planejamento (AP), regiões político-administrativas que correspondem, como unidade organizacional, aos distritos sanitários. As AP mantêm em comum, além da proximidade geográfica, o perfil sócioeconômico e de acesso aos serviços de saúde.

A AP 1 configura a zona mais antiga e central da cidade. As AP 2.I e 2.II correspondem respectivamente à Zona Sul e Zona Norte, tradicionalmente mais ricas. As AP 3 (3.I, 3.II e 3.III) compreendem os subúrbios da Central e Leopoldina, linhas férreas que cruzam a cidade, além da Ilha do Governador. As AP 4, 5.I, 5.II e 5.III correspondem à Zona Oeste, de ocupação mais recente. As AP 1, 2 e 3 correspondem a cerca de um quarto do território, apresentam alta densidade populacional e são dotadas de infra-estrutura urbana básica. A AP 3 responde por cerca da metade dos habitantes das favelas/setores subnormais de todo o município. As AP 4 e 5 ocupam em torno de $3 / 4$ do território, apresentam baixa densidade e carência de infra-estrutura básica. A AP 2.1 apresenta o nível de renda mais elevado e a Zona Oeste o mais baixo 24.

Toda a análise estatística foi feita usandose o programa R (R Foundation for Statistical Computing, Viena, Áustria; http://www.R-project.org), pacote estatístico de domínio público, e os mapas temáticos realizados no programa MapInfo (MapInfo Corporation, Troy, Estados Unidos). 


\section{Resultados}

No ano 2000 ocorreram no Município do Rio de Janeiro 4.015 óbitos por infarto agudo do miocárdio, destes, $9 \%$ de pessoas não-residentes na cidade. Dentre os óbitos em que esse município era o local de residência e também de ocorrência (3.651), mais da metade aconteceu em hospital $(58,1 \%)$.

A taxa bruta de mortalidade por infarto agudo do miocárdio para o município como um todo foi de 62,4 por 100 mil habitantes (mediana entre bairros de 59,8). Os homens apresentaram taxas mais elevadas do que as mulheres $(75,8$ e 50,7 por 100 mil habitantes, respectivamente). Cerca de $42 \%$ dos óbitos masculinos por infarto agudo do miocárdio ocorreram antes dos 65 anos de idade, entre as mulheres essa proporção foi mais baixa $(22,7 \%)$. A idade mediana de morte por infarto agudo do miocárdio foi de 67 anos para os homens e 75 para as mulheres. A distribuição da taxa de mortalidade por infarto agudo do miocárdio mostrou uma grande variabilidade entre os bairros $(0 \mathrm{a}$ 1315,79 por 100 mil habitantes), com um pequeno número de áreas com valores excessivamente altos.

Ao analisar os óbitos por causas mal definidas no Município do Rio de Janeiro em 2000, observou-se uma proporção elevada desse subgrupo em relação ao conjunto de causas de morte: 10,7\% (5.304 óbitos). Dos óbitos mal definidos, 71,6\% ocorreram em unidade hospitalar. Verificou-se uma concentração desse grupo de causas entre os maiores de 29 anos $(87,4 \%)$ e no sexo masculino $(52,8 \%)$. Os bairros apresentam uma grande variação em relação à proporção de causas mal definidas (0 a cerca de 50\%).

A distribuição espacial da razão não padronizada de mortalidade por infarto agudo do miocárdio, por bairro, configurou três aglomerados espaciais bem distintos (Figura 1). O primeiro, constituído por bairros que apresentaram um excesso de mortalidade por infarto agudo do miocárdio, concentra-se na região central do município, na Zona Sul e em parte da Zona Norte. O segundo, localizado no subúrbio da Leopoldina, também concentrou alta mortalidade, mas é mais disperso. Já o terceiro, composto por bairros localizados na Zona Oeste, agrega áreas que apresentaram baixa mortalidade. Nota-se que o padrão espacial com a estimativa bayesiana permaneceu o mesmo, no entanto há uma suavização das taxas.

A existência de autocorrelação espacial foi testada sobre as taxas não suavizadas por meio do índice I de Moran que, apesar de não ter sido alto $(0,0929)$, foi estatisticamente significa- tivo ( $p=0,0365$ ), indicando dependência espacial. Isto é, o teste confirma os achados de aglomerados espaciais de mortalidade por infarto agudo do miocárdio visualizados na Figura 1.

Após o ajustamento da RMP por infarto agudo do miocárdio por idade e sexo, o aglomerado que abrange a Zona Sul da cidade e alguns bairros da AP 2 (Tijuca, Grajaú), identificado na Figura 1, desapareceu e o localizado no subúrbio da Leopoldina se apresentou ainda mais disperso (Figura 2). Porém, o localizado na Zona Oeste da cidade se manteve. Quatro bairros se destacaram pelo excesso de mortalidade neste mapa temático: Saúde, Bonsucesso, Cidade Universitária e Vila da Penha.

Com a padronização por idade e sexo, a autocorrelação espacial identificada anteriormente desaparece, tendo o índice de Moran I $(0,0114)$ deixado de ser estatisticamente significativo ( $\mathrm{p}=0,5364$ ).

A distribuição espacial da proporção de óbitos por causas mal definidas, já suavizada por meio do modelo bayesiano empírico, mostrou uma alta concentração de óbitos mal definidos em toda a Zona Oeste da cidade (Figura 3). Comparando-se a distribuição da RMP por infarto agudo do miocárdio com a distribuição da proporção de óbitos por causas mal definidas, usando-se a sobreposição dos dois padrões espaciais, observou-se que em algumas áreas de baixo e médio risco de mortalidade por infarto agudo do miocárdio, localizadas na Zona Oeste e no subúrbio da Leopoldina, se concentra uma alta proporção de óbitos por causas mal definidas.

Observam-se dois aglomerados na distribuição espacial dos óbitos por infarto agudo do miocárdio ocorridos fora do hospital (Figura 4). Esses aglomerados, localizados na região central da cidade e na Zona Sul, distinguem-se pelo excesso de óbitos por infarto agudo do miocárdio ocorridos em domicílio, via pública e estabelecimentos de saúde que não sejam hospitais, como por exemplo postos e centros de saúde.

Os hospitais que prestam assistência ao infartado se distribuem geograficamente de forma muito peculiar no Município do Rio de Janeiro e essa distribuição guarda relação com a natureza jurídica do hospital. Os hospitais privados concentram-se principalmente na Zona Sul, em parte da Zona Norte (Tijuca, Vila Isabel), na Barra da Tijuca e em bairros de melhor condição social de outras áreas da cidade (Méier, Ilha do Governador). Os hospitais particulares conveniados ao SUS localizam-se predominantemente no centro da cidade, Jacarepaguá, Olaria, Penha Circular e na Zona Oeste. Os hos- 

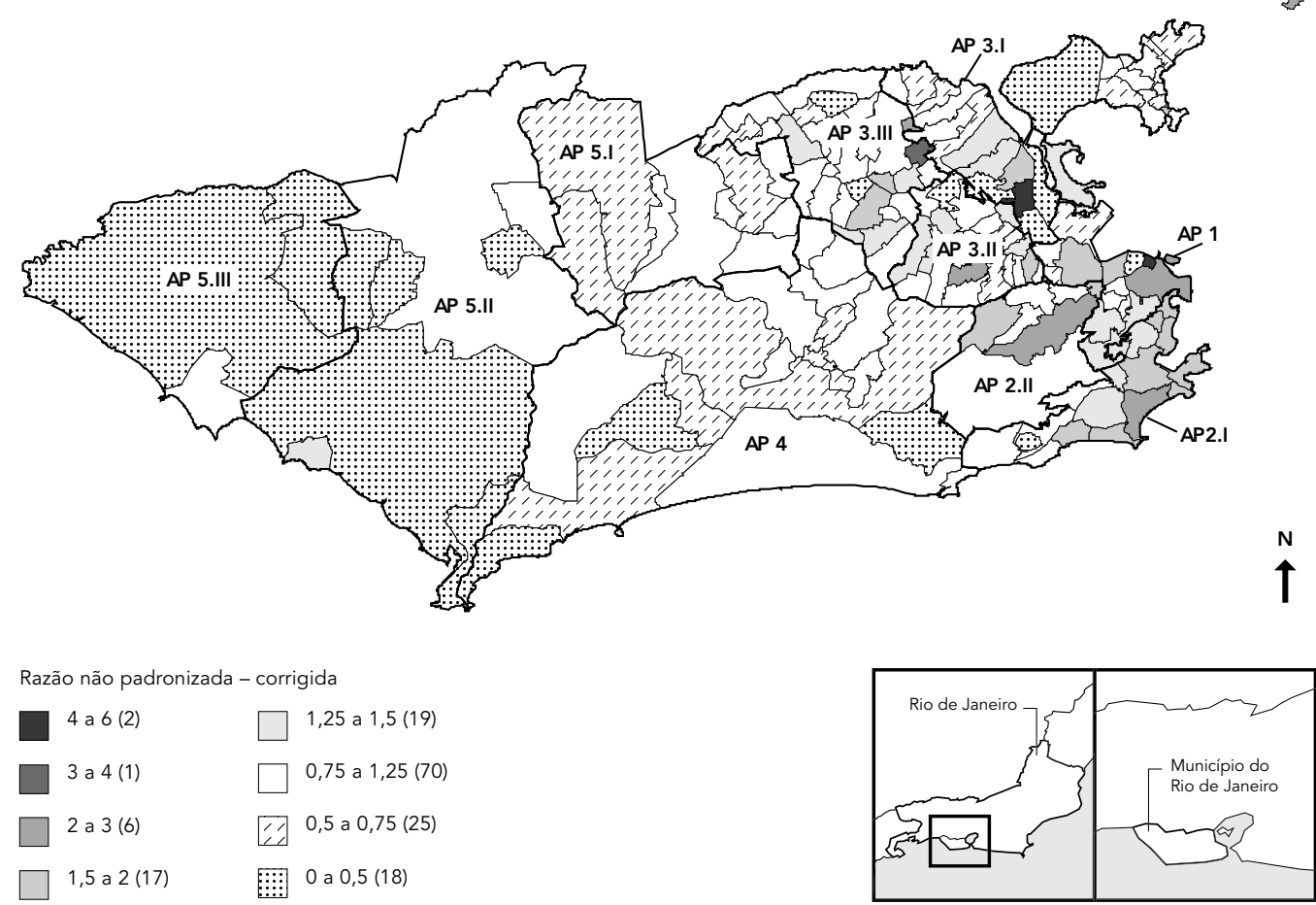

pitais estaduais estão localizados na Zona Oeste e na AP 3.I (Penha Circular) e os hospitais universitários na Cidade Universitária, Tijuca e Vila Isabel. Os hospitais federais estão distribuídos na região central da cidade e Bonsucesso. Os hospitais municipais localizam-se no centro da cidade, em parte da Zona Sul (Leblon, Ipanema, Lagoa, Botafogo), parte da Zona Norte (Andaraí, Méier), Ilha do Governador, Irajá, Guadalupe e Barra da Tijuca. Os hospitais filantrópicos estão concentrados no centro, na Tijuca e Usina.

A distribuição espacial suavizada da RMP, segundo vínculo do estabelecimento hospitalar onde o óbito ocorreu, mostra uma menor concentração de óbitos por infarto agudo do miocárdio em hospitais com financiamento do SUS nas áreas onde mora a população de melhor condição social (Zona Sul, Barra da Tijuca, Ilha do Governador) (Figura 5). A distribuição espacial da RMP mostra também que os óbitos por infarto agudo do miocárdio ocorridos no hospital obedecem a um padrão: têm sido atendidos próximo ao local de sua residência. No- ta-se uma clara atratividade do hospital com relação à população de sua cercania.

\section{Discussão}

A proporção de óbitos por infarto agudo do miocárdio de residentes fora do Município do Rio de Janeiro não foi alta (9\%), principalmente considerando-se que a cidade é referência para uma região metropolitana composta por 17 municípios (Duque de Caxias, Itaguaí, Mangaratiba, Nilópolis, Nova Iguaçu, São Gonçalo, Itaboraí, Magé, Maricá, Niterói, Paracambi, Petrópolis, São João de Meriti, Japeri, Queimados, Belford Roxo e Guapimirim, incluindo a capital do estado), com uma população em 2000 em torno de 14 milhões de habitantes 21 .

A elevada proporção observada de óbitos precoces (abaixo de 65 anos) reflete, de um lado, a alta concentração da população nas faixas etárias mais jovens e, de outro, um risco elevado de morte por infarto agudo do miocárdio no município. Essa magnitude da taxa de 


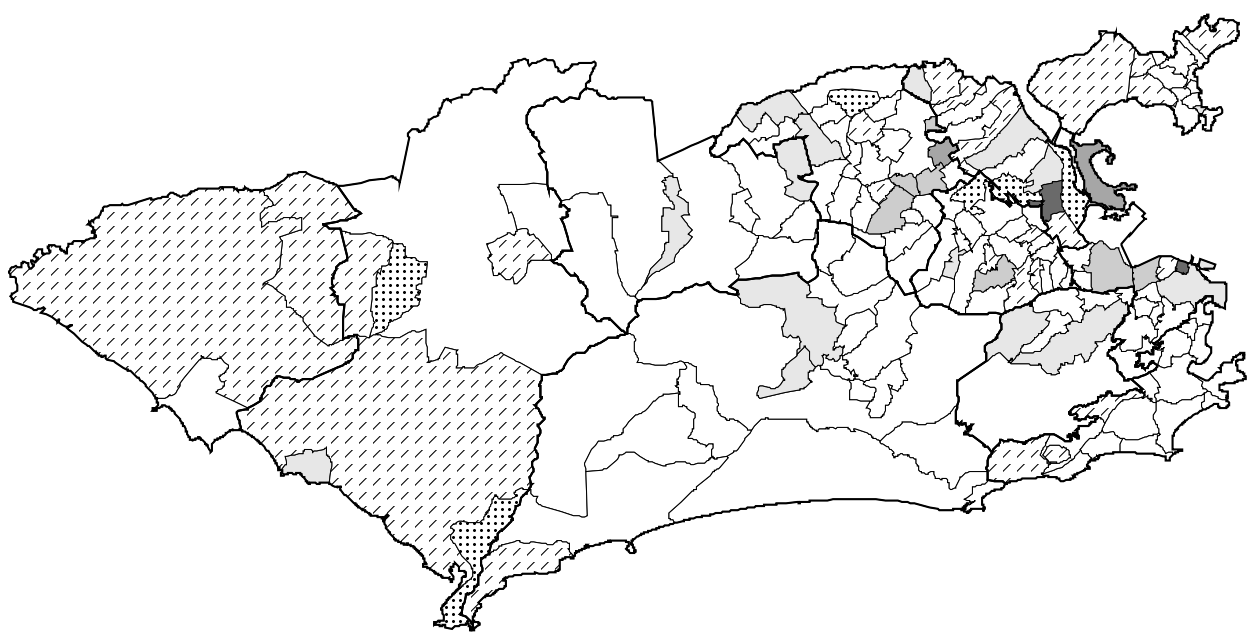

RMP - alisamento bayesiano
$\begin{array}{llll}3 \text { a } 4 \text { (2) } & \\ \square & \text { a } 3(2) & 0,75 \text { a } 1,25(100) \\ \square & 1,5 \text { a } 2(8) & 0,5 \text { a } 0,75(25) \\ \square & 1,25 \text { a } 1,5(15)\end{array}$

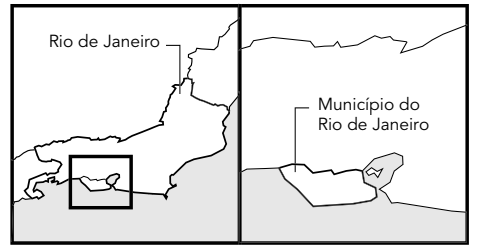

mortalidade no grupo das doenças isquêmicas do coração se compara àquela observada em outras capitais brasileiras 5,6. Considerou-se também elevada a proporção de óbitos por causas mal definidas no Município do Rio de Janeiro, que se mantém entre as cinco primeiras causas de óbito nesse município desde a última década. A elevação das causas mal definidas no Estado do Rio de Janeiro, em especial do código R99 que corresponde à causa indeterminada, coincide com a publicação da Resolução SES n. 55025 de 23 de janeiro de 1990. Essa resolução prevê que, esgotadas todas as tentativas no sentido de determinar a causa básica de morte (e não havendo suspeita de óbito por causa violenta), o óbito seja declarado como decorrente de "causa indeterminada". Assim, a partir de 1990, a região metropolitana do Rio de Janeiro apresentou um aumento significativo na proporção de causas mal definidas: mais de $200 \%$. Essa proporção que se mantinha em torno de 2,2 até 1989 chegou a 10,7 em 2000. Nesse aspecto, a ausência de um Serviço de Verificação de Óbitos representou um ônus em relação à qualidade das estatísticas de mortalidade no município. No mesmo período, outras regiões metropolitanas do Brasil (Belém, Fortaleza, Recife, Salvador, Belo Horizonte, São Paulo, Curitiba e Porto Alegre) apresentaram uma redução na proporção dessas causas 26,27.

Esse padrão de subnotifcação prejudica de um modo geral a análise das principais causas de mortalidade. No caso específico do infarto agudo do miocárdio a questão merece destaque, uma vez que se passou de uma situação de sobrenotificação de óbitos, observada até 1989, para um padrão inverso de subnotificação. Estudo voltado para a investigação dos óbitos por causas mal definidas, realizado pela equipe da Gerência de Informações Epidemiológicas da SMS-RJ, mostrou que 13,5\% dos óbitos recuperados pertenciam ao grupo de doenças do aparelho circulatório 28. Pesquisa realizada por Teixeira 29 recuperou as informações relativas aos óbitos por causa mal definida ocorridos no Estado do Rio de Janeiro em 1998, por meio do processo de linkagem entre as bases de dados do SIM e do Sistema de Informação 


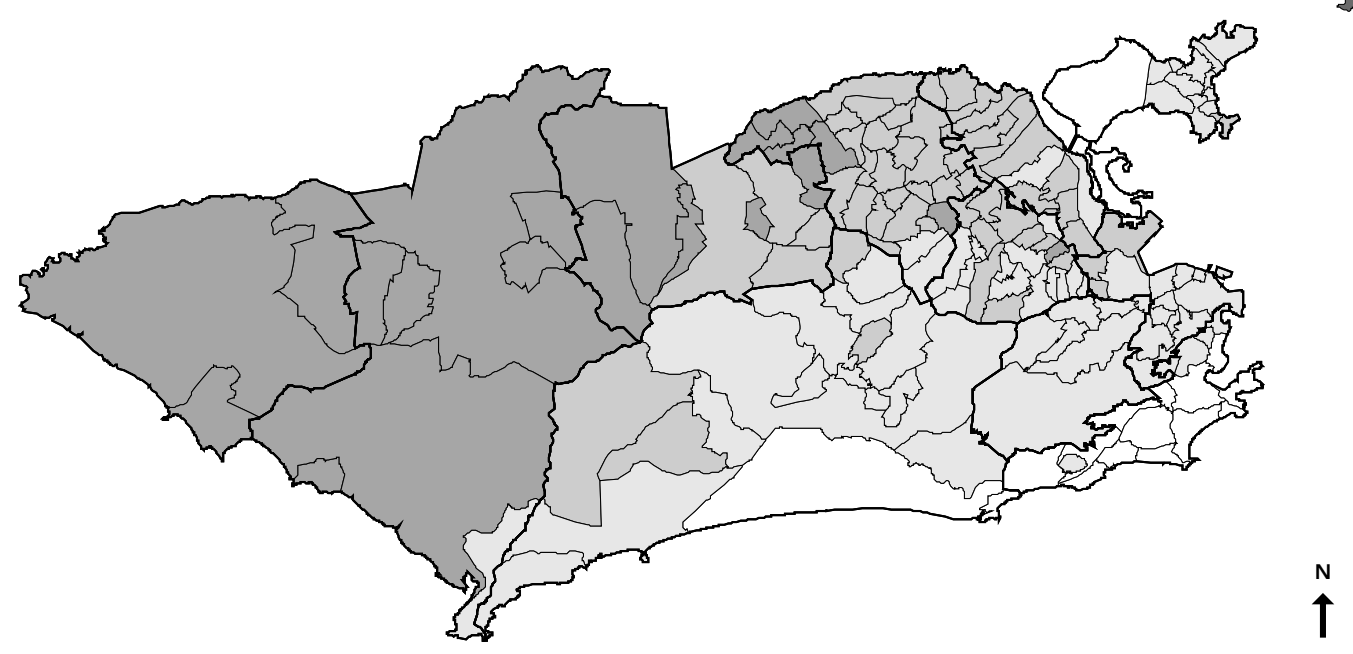

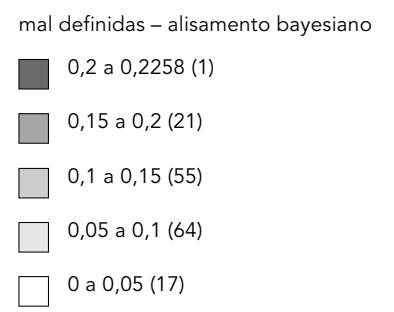

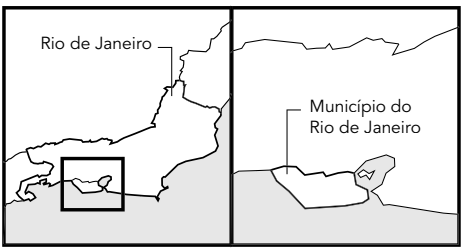

Hospitalar (SIH-SUS). Este estudo mostrou que 25\% das internações, entre os casos localizados em que o intervalo entre a última internação e o óbito foi inferior a 48 horas (575 óbitos), pertenciam ao grupo de doenças do aparelho circulatório 29. Estudo realizado por Oliveira et al. 30 mostrou a necessidade de correção dos óbitos por doença isquêmica do coração que vêm sendo subnotificados em função da alta proporção de óbitos por causas mal definidas.

A distribuição espacial dos óbitos por infarto agudo do miocárdio observada no Município do Rio de Janeiro é heterogênea e o padrão espacial identificado está associado a um forte gradiente social. Esse padrão em mosaico é compatível com os contrastes sociais observados no município e descritos em outros estudos 2,18.

O Rio de Janeiro agrega um padrão extremamente heterogêneo no que se refere ao processo de ocupação e à distribuição geográfica da população. Áreas remanescentes de floresta se alternam com áreas densamente povoadas; áreas consideradas nobres coexistem, lado a lado, com os estratos mais pobres da população. Essa composição traz para a cidade uma organização sócio-econômica espacial marcada por contrastes.

O padrão de sub-risco de mortalidade por infarto agudo do miocárdio observado na Zona Oeste (principalmente AP 5.I e 5.III) não condiz com o perfil de desigualdade social e de acesso aos serviços de saúde observado na área. Acredita-se que o risco de morrer por infarto agudo do miocárdio foi subestimado em função da alta proporção de óbitos por causa mal definida nessa área.

O mapeamento da mortalidade por causas mal definidas mostrou que a subnotificação de óbitos por infarto agudo do miocárdio se concentra principalmente em áreas socialmente desfavorecidas. Neste estudo, essas causas estiveram concentradas na Zona Oeste (AP 5) e em parte do subúrbio da Central e Leopoldina (AP 3.III), áreas mais distantes do centro urba- 


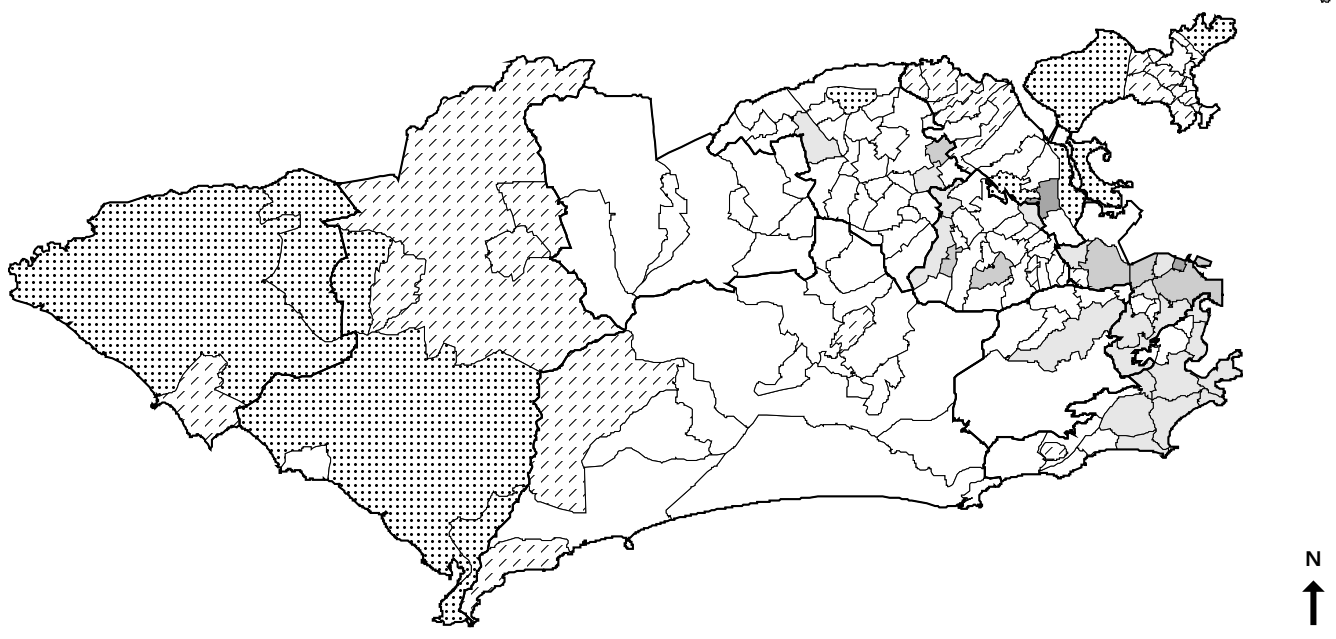

$$
\begin{aligned}
& \text { RMP - óbitos fora do hospital } \\
& \begin{array}{ll}
2 \text { a } 3 \text { (3) } & 0,5 \text { a } 0,75(24) \\
\square & 1,5 \text { a } 2(6) \\
\square & 1,25 \text { a } 1,5(20) \\
\square & 0,75 \text { a } 1,25(94)
\end{array}
\end{aligned}
$$

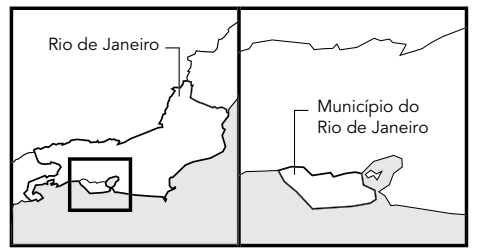

no da cidade e de maior carência de serviços. Os óbitos por causas mal definidas, mais do que identificar a qualidade das informações nas DO, apontam para deficiências no acesso aos serviços de saúde e na qualidade da atenção.

A correlação espacial inicialmente observada na distribuição da mortalidade por infarto agudo do miocárdio mostrou-se condicionada à estrutura etária dos bairros. Ao contrário de outras cidades, onde se verifica uma organização espacial que concentra no centro áreas consideradas nobres, verifica-se no Município do Rio de Janeiro um padrão em mosaico. Essa diversidade, onde coexistem lado a lado perfis sócio-econômicos muito distintos, e a unidade de análise estudada (bairros) explicam, em parte, a ausência de autocorrelação.

Este estudo evidencia que os infartados tendem a ser atendidos próximo ao local de residência, o que pode ser explicado pelo fato do infarto ocorrer mais freqüentemente nas primeiras horas da manhã e durante a noite 31 . Deve ser considerada ainda a alta concentração de óbitos acima de 70 anos (52,6\%), idade em que muitos já estão aposentados. Considerando que os hospitais mostraram exercer uma atratividade em relação à população de sua cercania, o local de residência é uma informação relevante para identificação da população de referência.

Considerando que o intervalo de tempo decorrido entre o início dos sintomas e a admissão hospitalar é importante para o prognóstico pós-infarto agudo do miocárdio, a distância em relação aos serviços de saúde tem um peso fundamental na sobrevida do paciente. Apesar do Município do Rio de Janeiro apresentar uma das maiores redes hospitalares vinculadas ao SUS do Brasil, verifica-se que a distribuição geográfica destas unidades é extremamente desigual, com elevada concentração na região central da cidade. Essa distribuição está relacionada ao processo de ocupação e à própria trajetória política da cidade.

Cabe destacar que a produção de mapas temáticos exige extremo cuidado, especialmente nos casos de populações com valores reduzidos nas medidas de interesse. O processo de 


\section{Figura 5}

Distribuição espacial da razão de mortalidade por infarto agudo do miocárdio padronizada por idade e sexo. Bairros do Município do Rio de Janeiro, Brasil, 2000.

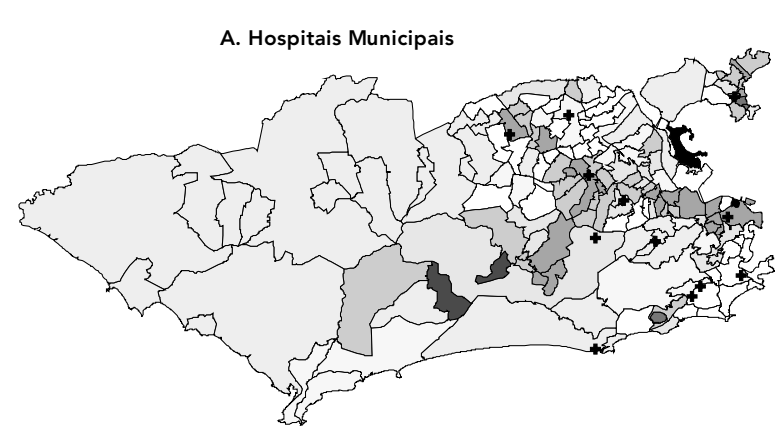

RMP - Hospital Municipal

\begin{tabular}{|c|c|c|}
\hline 6 a $40(2)$ & 2 a $3(20)$ & 0,75 a $1,25(38)$ \\
\hline 4 a 6 (2) & 1,5 a $2(27)$ & 0,5 a $0,75(17)$ \\
\hline 3 a $4(4)$ & 1,25 a $1,5(16)$ & 0 a 0,5 (32) \\
\hline
\end{tabular}

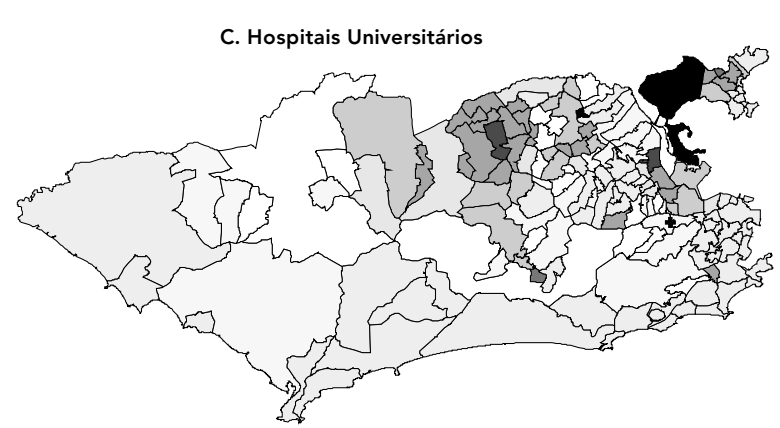

RMP - Hospital Universitário
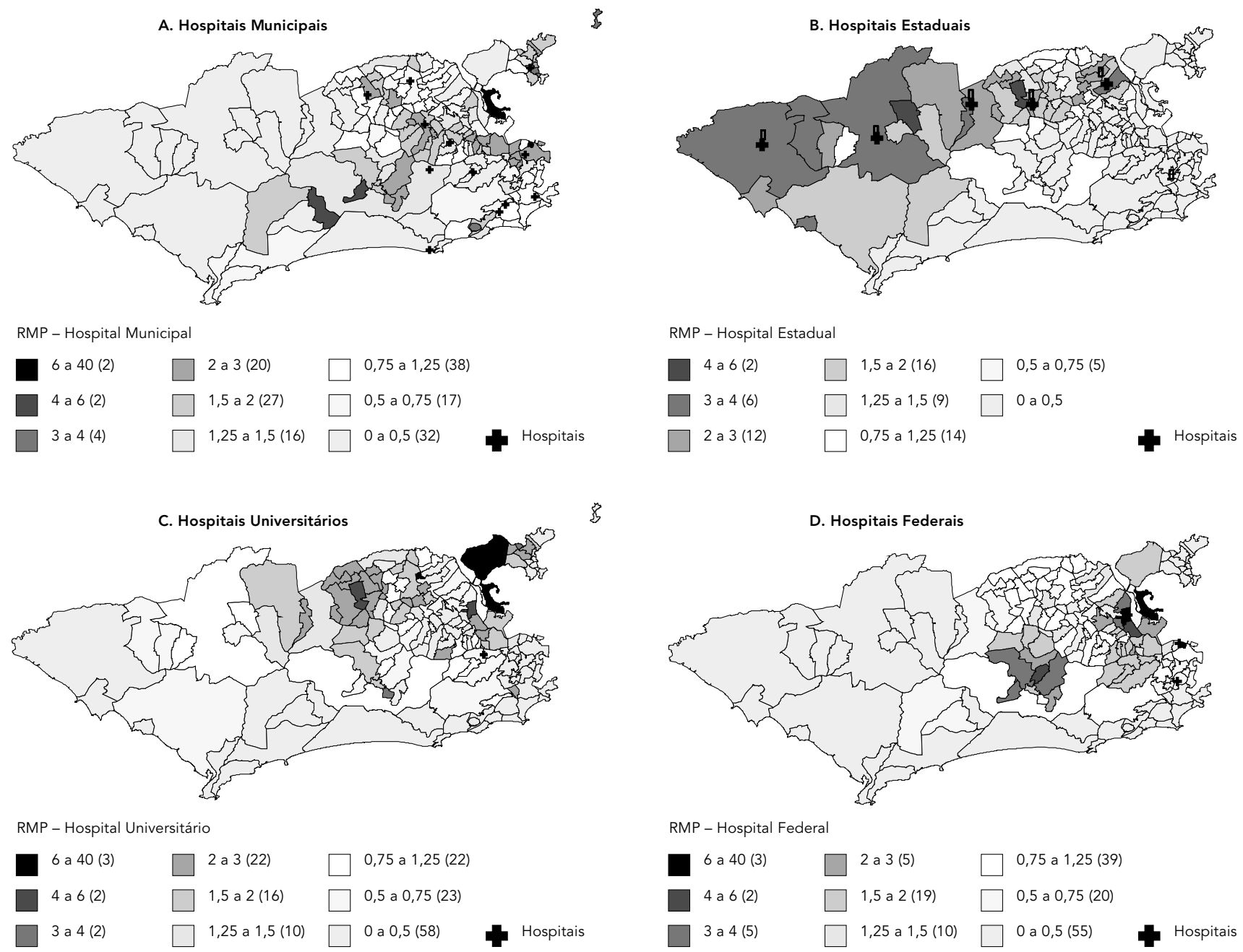

RMP - Hospital Federal

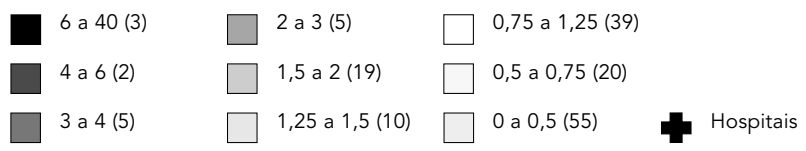

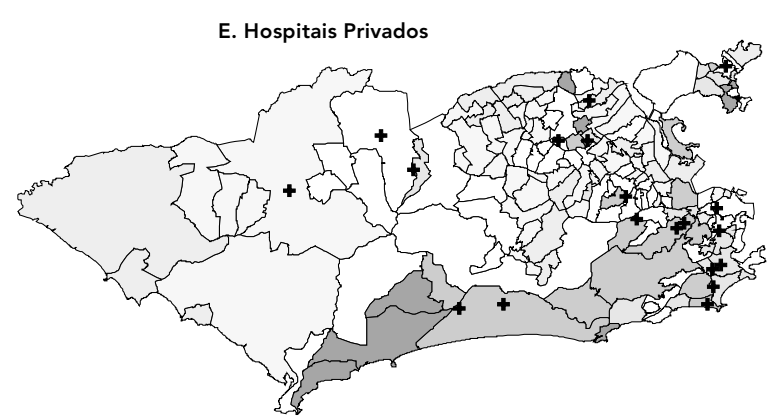

RMP - Hospital Privado
6 a 40 (1)
$\square \quad 1,25$ a $1,5(18)$
0 a $0,5(20)$
2 а 3 (8)
$\square 0,75$ a $1,25(54)$
1,5 a 2 (14)
0,5 a $0,75(43)$

C. Hospitais

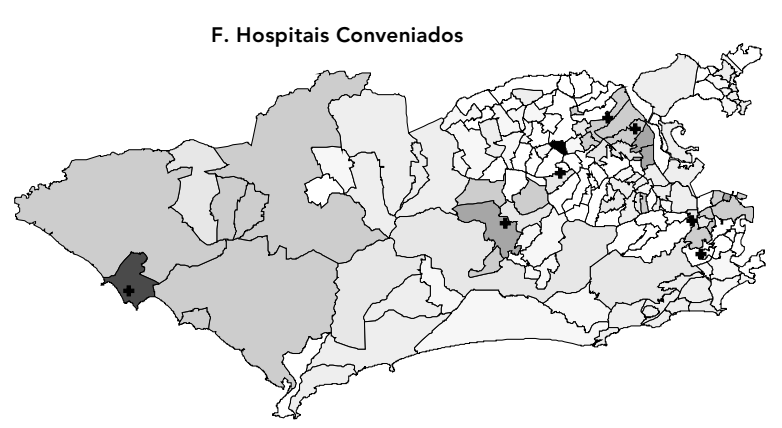

RMP - Hospitais Conveniados
D 6 a 40 (1)
$\square \quad 1,5$ a $2(20)$
$\square \quad 1,25$ a $1,5(13)$
$\square \quad 0,5$ a 0,75 (29)
$\square$ a 6 (1)
0,75 a 1,25 (54)
$\square 2$ a $3(4)$ 
suavização utilizado neste estudo permitiu estimar melhor as RMP, utilizando informações de unidades espaciais vizinhas. Recomenda-se a utilização desse procedimento uma vez que reduz a influência da flutuação aleatória das medidas, permitindo a produção de mapas mais confiáveis e a visualização de áreas que apresentam risco elevado. Esse tipo de procedimento, no entanto, ainda é de difícil aplicação nos ambientes de SIG e nos programas estatísticos. Contudo, dado a sua importância, o processo de suavização local começa a ser mais utilizado 22,32 e deverá estar, em breve, disponível em uma das interfaces de menu do TerraView (Instituto Nacional de Pesquisas Espaciais, São José dos Campos, Brasil; http://www. dpi.inpe.br/terraview/index.php), aplicativo geográfico do programa de domínio público TerraLib desenvolvido por meio de projeto interinstitucional coordenado pela Divisão de Processamento de Imagens (DPI) do Instituto Nacional de Pesquisas Espaciais (INPE).

Um problema relacionado à qualidade dos dados deve ser destacado, pois pode ter interferido nos resultados deste estudo: a localiza- ção do bairro de residência. Apesar da baixa proporção de óbitos com endereço ignorado $(0,5 \%)$, acredita-se que há problemas na qualidade do preenchimento desse campo, como já relatado por Santos \& Noronha 19. Bairros maiores e/ou mais conhecidos tendem a ser mais citados pela população, tipo de problema relacionado ao georreferenciamento dos casos. Esse fato pode resultar na subenumeração de óbitos em algumas áreas. Considera-se a possibilidade de subenumeração de óbitos em alguns dos bairros criados mais recentemente (Rocinha, Complexo da Maré, Complexo do Alemão, Parque Columbia).

Somando-se a outros estudos voltados para a análise de agravos à saúde no Município do Rio de Janeiro, este estudo mostra que a incorporação do elemento geográfico, por meio da sua importante contribuição na identificação de áreas e situações de risco, abre a possibilidade do redirecionamento de ações de saúde, principalmente em áreas onde se verifica maior exclusão social. Para tanto, é fundamental dispor de sistemas de informação de boa qualidade, ágeis e integrados.

\section{Resumo}

O objetivo deste estudo é analisar a distribuição espacial da mortalidade por infarto agudo do miocárdio no Município do Rio de Janeiro, Brasil. Foram analisados dados sobre mortalidade por infarto agudo do miocárdio ocorrido em 2000, por meio do Sistema de Informação de Mortalidade. Utilizou-se o modelo bayesiano empírico de suavização a fim de minimizar a variabilidade aleatória dos coeficientes de mortalidade associada ao tamanho das unidades geográficas de análise. A distribuição dos óbitos por infarto agudo do miocárdio na cidade é heterogênea e obedece a um padrão espacial associado a um forte gradiente social. $O$ padrão de sub-risco de mortalidade por infarto agudo do miocárdio observado na Zona Oeste não condiz com o perfil de desigualdade social e de acesso aos serviços de saúde observado na área. Acredita-se que o risco de morrer por infarto agudo do miocárdio foi subestimado em função da alta proporção de óbitos por causa mal definida na área. O padrão espacial de mortalidade apresentou uma concentração do risco de morrer de infarto nas áreas mais pobres da cidade. As diversas unidades de saúde apresentam áreas de influência para o atendimento ao infarto agudo do miocárdio.

Infarto do Miocárdio; Análise Espacial; Sistemas de Informação; Acesso aos Serviços de Saúde 


\section{Colaboradores}

E. C. P. Melo participou da concepção da pesquisa, análise e processamento dos dados e redação do artigo. M. S. Carvalho e C. Travassos contribuíram na concepção da pesquisa, análise dos dados e redação do artigo.

\section{Referências}

1. Mansur AP, Favarato D, Souza MFM, Avakian SD, Aldrighi JM, César LAM, et al. Trends in death from circulatory diseases in Brazil between 1979 and 1996. Arq Bras Cardiol 2001; 76:504-10.

2. Souza MC. Modelos de regressão ecológica: uma aplicação em doença isquêmica do coração, Rio de Janeiro, 1991 [Dissertação de Mestrado]. Rio de Janeiro: Escola Nacional de Saúde Pública, Fundação Oswaldo Cruz; 2000.

3. Kirchhoff M, Davidsen M, Bronnum-Hansen B, Schnack H, Eriksen LS, Madsen M. Incidence of myocardial infarction in the Danish MONICA population 1982-1991. Int J Epidemiol 1999; 28:211-8.

4. Rosamond WD, Chambless LE, Folsom AR, Cooper LS, Conwill DE, Clegg L, et al. Trends in the incidence of myocardial infarction and in mortality due to coronary heart disease, 1987 to 1994 . N Engl J Med 1998; 339:861-7.

5. Lotufo PA. Mortalidade precoce por doenças do coração no Brasil. Comparação com outros países. Arq Bras Cardiol 1998; 70:321-5.

6. Chor D, Fonseca MJM, Andrade CR, Waissman W, Lotufo PA. Doenças cardiovasculares. Mortalidade precoce no Brasil. Arq Bras Cardiol 1995; 64: 15-9.

7. Gatrell A, Gillian L, Chapple A, Horsley S, Smith $M$. Variations in use of tertiary cardiac services in part of North-West England. Health Place 2002; 8:147-53.

8. Silva MAD, Sousa AGM, Schargodsky H. Fatores de risco para infarto do miocárdio no Brasil. Estudo FRICAS. Arq Bras Cardiol 1998; 71:667-75.

9. Tydén P, Handen O, Engström G, Hedblad B, Janzon L. Myocardial infarction in an urban population: worse long term prognosis for patients from less affluent residential areas. J Epidemiol Community Health 2002; 56:785-90.

10. Souza MFM, Timerman A, Serrano Jr. CV, Santos $\mathrm{RD}$, Mansur AP. Trends in the risk of mortality due to cardiovascular diseases in five brasilian geographic regions from 1979 to 1996 . Arq Bras Cardiol 2001; 77:569-75.

11. Wamala SP, Lynch J, Kaplan GA. Women's exposure to early and later life socioeconomic disadvantage and coronary heart disease risk: the Ltockholm Female Coronary Risk Study. Int J Epidemiol 2001; 30:275-84.

\section{Agradecimentos}

Aos organizadores da III Oficina de Publicação de Artigos, promovida pelo Programa de Pós-graduação da Escola Nacional de Saúde Pública Sergio Arouca da Fundação Oswaldo Cruz, em especial ao Prof. Dr. Reinaldo Souza dos Santos.

12. Lang $\mathrm{T}$, Ducimetière $\mathrm{P}$, Arveiler $\mathrm{D}$, Amuoyel $\mathrm{P}$, Ferrières $\mathrm{J}$, Ruidavets $\mathrm{JB}$, et al. Is hospital care involved in inequalities in coronary heart disease mortality? Results from the French WHO-MONICA Project in men aged 30-64. J Epidemiol Community Health 1998; 52:665-71.

13. Mackenbach JP, Looman CWN, Kunst AE. Geographic variation in the onset of decline of male ischemic heart disease mortality in the Netherlands. Am J Public Health 1989, 79:1621-7.

14. Alter DA, Naylor CD, Austin P, Chan BTB, Tu JV. Geography and service supply do not explain socioeconomic gradients in angiography use after acute myocardial infarction. CMAJ 2003; 168:261-4.

15. Philbin EF, McCullough PA, DiSalvo TF, Dee GW, Jenkins PL, Weaver D. Socioeconomic status in an important determinant of the use of invasive procedures after acute myocardial infarction in New York State. Circulation 2000; 102 Suppl 3:III107-15.

16. Campos TP, Carvalho MS, Barcellos CC. Mortalidade infantil no Rio de Janeiro, Brasil: áreas de risco e trajetória dos pacientes até os serviços de saúde. Rev Panam Salud Publica 2000; 8:164-71.

17. Szwarcwald CL, Bastos FI, Esteves MA, Andrade CLT, Paez MS, Medici EV, et al. Desigualdade de renda e situação de saúde: o caso do Rio de Janeiro. Cad Saúde Pública 1999; 15:15-28.

18. Szwarcwald CL, Bastos FI, Barcellos C, Pina MF, Esteves MAP. Health conditions and residential concentration of poverty: a study in Rio de Janeiro, Brazil. J Epidemiol Community Health 1999; 54:530-6.

19. Santos S, Noronha CP. Padrões espaciais de mortalidade e diferenciais sócio-econômicos na Cidade do Rio de Janeiro. Cad Saúde Pública 2001; 17:1099-110.

20. Birkhead J, Goldacre M, Manson A, Wilkinson E, Armess M, Cleary R, editors. Health outcome indicators: myocardial infarction. Oxford: National Centre for Health Outcomes Development; 1999.

21. Instituto Brasileiro de Geografia e Estatística. Censo demográfico de 2000. Resultados do universo. http://www.sidra.ibge.gov.br/bda/pesquisas/ cdru/default.asp (acessado em 04/Mar/2004).

22. Assunção RM, Barreto SM, Guerra HL, Sakurai E. Mapas de taxas epidemiológicas: uma abordagem Bayesiana. Cad Saúde Pública 1998; 14:713-23. 
23. Cliff AD, Ord JK. Spatial processes: models and applications. London: Pion; 1981.

24. Bastos-Cezar P. Evolução da população de favelas na Cidade do Rio de Janeiro: uma reflexão sobre os dados mais recentes. Rio de Janeiro: Instituto Pereira Passos, Secretaria Municipal de Urbanismo; 2002. (Coleção Estudos da Cidade).

25. Secretaria de Estado de Saúde do Rio de Janeiro. Resolução 550/1990, de 23 de janeiro de 1990. Diário Oficial do Estado do Rio de Janeiro 1990; 23 jan.

26. Aidar T. Registro de óbitos por causa mal definida: atenção especial para o caso da população feminina. Brasil 1979 a 1995. Anais do XII Encontro de Estudos Populacionais da ABEP. v. 1. http:// www.abep.nepo.unicamp.br/docs/anais/pdf/200 0/Todos/Registro\%20de\%20abitos\%20com\%20C ausa\%20Mal\%20Definida.pdf (acessado em 30/ Set/2003).

27. Paes NA. A geografia da mortalidade por causas dos idosos no Brasil. Anais do XII Encontro de Estudos Populacionais da ABEP. v. 1. http://www. abep.nepo.unicamp.br/docs/anais/pdf/2000/Todos/saut18_3.pdf (acessado em 30/Set/2003).

28. Caridade MC, Campos TP, Serafim FC, Bellizzi ALM, Simplício AMB, Silva RI, et al. Causas mal definidas de morte: recuperação de informações na Cidade do Rio de Janeiro. Rio de Janeiro: Gerência de Informações Epidemiológicas, Coorde- nação de Programas de Epidemiologia, Superintendência de Saúde Coletiva, Secretaria Municipal de Saúde do Rio de Janeiro; 2003.

29. Teixeira CLS. Relacionamento e reclassificação dos óbitos de causa mal definida com as Autorizações de Internação Hospitalar no Sistema Único de Saúde, no Rio de Janeiro [Dissertação de Mestrado]. Rio de Janeiro: Universidade Federal do Rio de Janeiro; 2004.

30. Oliveira GMM, Souza-e-Silva NA, Klein CH. Mortalidade por doenças isquêmicas do coração, doenças cerebrovasculares e causas mal definidas nas regiões de saúde do Estado do Rio de Janeiro, no período de 1980 a 2000. Rev SOCERJ 2003; 16 Suppl A:78.

31. Maemura K, De La Monte SM, Chin MT, Layne MD, Hsieh CM, Yet SF, et al. CLIF, a novel cyclelike factor, regulates the circadian oscillation of plasminogen activator inhibitor-1 gene expression. J Biol Chem 2000; 275:36847-51.

32. Souza WV, Barcellos CC, Brito AM, Carvalho MS, Cruz OG, Albuquerque MFM, et al. Aplicação de modelo bayesiano empírico na análise espacial da ocorrência de hanseníase. Rev Saúde Pública 2001; 35:474-80.

Recebido em 24/Jun/2005

Versão final reapresentada em 05/Out/2005 Aprovado em 17/Out/2005 